\title{
Obstacle Emergence Risk and Road Patrol Policy
}

\author{
Kiyoshi Kobayashi ${ }^{1}$ and Kiyoyuki Kaito ${ }^{2}$ \\ ${ }^{1}$ Graduate School of Management, Kyoto University, Yoshida-Honmachi, Sakyo-ku, Kyoto 606-8501, Japan \\ ${ }^{2}$ Department of Civil Engineering, Osaka University, 2-1 Yamada-oka, Suita, Osaka 565-0871, Japan
}

Correspondence should be addressed to Kiyoshi Kobayashi, kkoba@psa.mbox.media.kyoto-u.ac.jp

Received 1 August 2011; Accepted 17 September 2011

Academic Editor: Ram M. Pendyala

Copyright ( 2012 K. Kobayashi and K. Kaito. This is an open access article distributed under the Creative Commons Attribution License, which permits unrestricted use, distribution, and reproduction in any medium, provided the original work is properly cited.

The authors model the emergence processes of road obstacles, such as fallen objects on roads, the deformation and destruction of pavements, and the damage and destruction of road facilities, as counting processes. Especially, in order to take into account the heterogeneity of the emergence risk of a variety of road obstacles, the authors model a mixture Poisson process in which the arrival rate of road obstacles is subject to a probability distribution. In detail, the authors formulate a Poisson-Gamma model expressing the heterogeneity of the arrival rate as a Gamma distribution and formulate the management indicator of the emergence risk of road obstacles. Then, a methodology is developed in order to design a road patrol policy that can minimize the road obstacle risk with a limited amount of budget. Furthermore, the authors empirically verify that it is possible to design road patrol policy based on the emergence risk of actual road obstacles with the proposed methodology, by studying the cases of the application of the methodology to general national roads.

\section{Introduction}

Road administrators must make efforts to keep roads in a sound condition. Particularly, fallen objects on roads and the deformation or destruction of pavements have the risk of inducing a vehicle-damaging accident. Accordingly, road administrators are required to patrol roads constantly and remove fallen objects on roads and repair the deformation of pavements. On the other hand, amid the retrenched finance due to the dwindling birthrate and aging society, it is imperative to streamline administrative tasks also in the field of the maintenance and repair of road facilities. In the road administration costs, the proportion of road patrol costs is not low, and so it is necessary to discuss road patrol methods while considering efficiency as well as safety.

In general, road patrol is carried out at certain intervals. Therefore, the costs for road patrol are fixed, no matter whether or not there are fallen objects or damages on roads. On the other hand, the probability of discovering a fallen object or damage on roads in a unit period varies significantly according to road sections. As the frequency of road patrol is increased, it is possible to respond more swiftly to the emergence of an event that would degrade the safety and traffic flow on roads, and the risk of leaving a road obstacle on a road for a long time. Meanwhile, frequent road patrol results in the increase in patrol expenditures and the augmentation of social costs. Like this, there is a trade-off relation between the obstacle emergence risk and the road patrol costs, and so road administrators need to set up a goal for controlling the obstacle emergence risk and then design road patrol policies so as to minimize the road patrol costs.

The emergence processes of road obstacles can be modeled as counting processes in which each event occurs randomly. In general, the emergence of fallen objects and the deformation and damage of pavements can be modeled as Poisson processes. However, Poisson processes are restricted by the assumption that the mean value is equal to its variance [1]. The actual emergence processes of road obstacles do not always have such a characteristic, and so more flexible modeling is necessary. In this circumstance, this paper proposes mixture Poisson processes in which it is possible to take into account the heterogeneity of the arrival rates of road obstacles [2]. In this proposal, arrival rates are expressed by a Poisson model, and the emergence risk of road obstacles is expressed by using the Poisson Gamma model in which the heterogeneity of the arrival rates is 
described by the Gamma distribution, and then risk control indicators are formulated. Furthermore, this paper proposes a methodology for designing a road patrol policy that can minimize the road obstacle risk with a limited amount of budget. Here, as the indicator for controlling the road obstacle risk, the authors focus on the number of road obstacles discovered during patrol (hereinafter called the number of road obstacles). In addition, as factors in the design of road patrol policy, this paper discusses the target road section for a single patrol (basic management section), the patrol frequency in each basic management section, and the road section where intensive patrol is conducted (intensive management section). However, the number of possible patrol policy designed by combing these factors is enormous, and many of them are not feasible. In this circumstance, this paper introduces a practical methodology for selecting road patrol policy that can reduce the road obstacle risk with a limited amount of budget among those suggested by road administrators.

\section{Basic Ideas of This Study}

2.1. Overview of Conventional Study. Road patrol is carried out with the purpose of removing obstacles left on roads and swiftly responding to the damage or destruction of road facilities and so forth. The emergence processes of such road obstacles are random phenomena, and such emergence processes can be modeled as probabilistic processes. Also in the field of civil engineering, there have been many researches that modeled random arrival events using Poisson processes [2]. In Poisson processes, the arrival rate of the analysistarget event is expressed by a deterministic constant, and so there is an advantage that its mathematical handling is easy. However, there are a variety of road obstacles, and it is not true that all events occur with the same arrival rate. In addition, Poisson processes are restricted by the assumption that the expectation of the number of road obstacles during a cycle period is equal to its variance. Especially, when the expectation of road obstacles is very small, the variance is also very small, and then the event of the emergence of a road obstacle becomes a rare phenomenon. Namely, there emerges an overdispersion problem that the variance of the number of road obstacles is larger than its expectation. In order to solve such an overdispersion problem, it is necessary to use the mixture Poisson processes that allow the heterogeneity of the arrival rate of road obstacles.

The research into mixture Poisson processes were pioneered by Fisher [3], and then various modifications were attempted $[4,5]$. Poisson processes have been applied to the evaluation of operational risk and accident risk. In general, the model structure for a mixture Poisson process becomes extremely complicated, because an event probability distribution and an event interval probability distribution are combined. However, the Poisson-Gamma model used in this study has the simplest model structure among mixture Poisson process models and has the advantage that it can express a model theoretically. Another advantage is that it is possible to readily obtain indicators for controlling the road

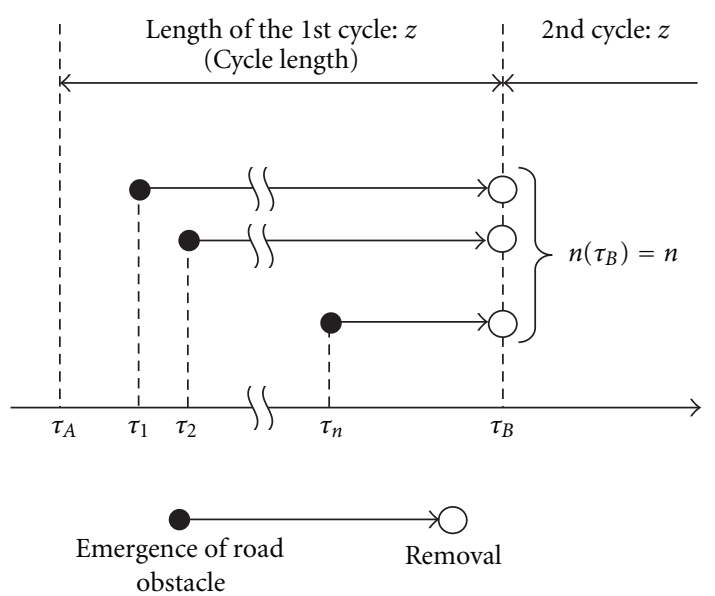

FIgURE 1: Emergence process of road obstacles.

obstacle risk. For these reasons, the Poisson-Gamma model is employed in this study, to describe the emergence processes of road obstacles.

The road patrol frequency is closely related to the obstacle emergence risk. By decreasing the road patrol frequency, it is possible to reduce the patrol costs. On the other hand, when road obstacles are ignored for a long time, the obstacle emergence risk augments, causing traffic accidents. Like this, there exists a trade-off relation between the patrol costs and the obstacle emergence risk, via the road patrol frequency. In this circumstance, this paper models the emergence processes of road obstacles as mixture Poisson processes and proposes a methodology for designing road patrol policy with the purpose of curtailing the patrol costs effectively. As far as the authors know, there have been no researches that discussed rational road patrol policy based on empirical measurements of road obstacles, except this study. In addition, the PoissonGamma model used in this study is the same as that proposed by previous studies $[6,7]$, although minor modifications are made. However, with regard to the risk control indicators proposed in this study, there have been no researches, as far as the authors know.

2.2. Road Patrol Scheme. Let us model the emergence processes of road obstacles. Firstly, a target road is divided into several road sections. Then, suppose that the time-series data about the frequency of the road obstacle emergence in each road section is available. As shown in Figure 1, road patrol is carried out in each road section at the same intervals along the time axis. Time $\tau$ represents actual time on a calendar. Hereinafter, actual time is called "time." At the times $\tau_{A}, \tau_{B}, \ldots$ in the figure, road patrol is started. The period from a certain patrol time to the next patrol time is called "cycle." The time interval between the contiguous patrol times is called "cycle length." The cycle length in the figure is $z$. When road patrol is conducted and a road obstacle is discovered, the obstacle is removed immediately. On the other hand, a road obstacle emerges at each of the times $\tau_{1}, \tau_{2}, \ldots$. However, road administrators cannot observe the time at which a road obstacle emerged. By conducting road 
patrol at time $\tau_{B}$, it is possible to observe the road obstacles that emerged during the cycle length $\left[\tau_{A}, \tau_{B}\right)$, as shown in Figure 1.

Here, suppose that a road obstacle follows the Poisson arrival with a certain arrival rate $\lambda$. In Figure 1, the number of road obstacles that emerged on a certain road section during the period from time $\tau_{A}$ to time $t$ is expressed by a counting process $n(t)$. Obviously, $n\left(\tau_{A}\right)=0$ at the initial time $\tau_{A}$. There emerged $n$ road obstacles by time $\tau_{B}$, and the $n+1$ th obstacle did not emerge. From time $\tau_{B}$, the second cycle starts with the initial time being $\tau_{B}$. In the case where a road obstacle follows the Poisson arrival, the time of the emergence of the first road obstacle in the second cycle does not depend on the time of the emergence of the last road obstacle in the first cycle. Namely, the emergence of a road obstacle is not dependent on the history of road obstacle emergence.

\section{Poisson Gamma Model}

3.1. Mixture Poisson Process. In Poisson processes, it is assumed that one kind of event occurs repeatedly with the same arrival rate. However, road obstacles include various kinds of fallen objects, road deformation, and damage and destruction of road facilities, and it is hard to believe that all of these obstacles emerge with the same arrival rate. It is rather appropriate to consider a phenomenon in which a variety of road obstacles emerge randomly. Suppose that many types of road obstacles emerge with different arrival rates and the arrival rate in a certain period is subject to a probability distribution. Namely, suppose that the arrival rates of road obstacles are subject to a probability distribution for each target road section. At the same time, suppose that road obstacles emerge in accordance with Poisson processes for each road section. The Poisson process in which arrival rates are subject to a probability distribution is called a "mixture Poisson process." By utilizing mixture Poisson processes, it is possible to remove the constraint condition that expectation is equal to variance in Poisson processes. Accordingly, it is possible to model a more flexible counting process. With such a mixture Poisson process, the heterogeneity of arrival rates is expressed by a Gamma distribution, and the event emergence is described by a Poisson process model. The Poisson-Gamma model has the simplest model structure among mixture Poisson models, and this model can be expressed theoretically. Furthermore, this model has a characteristic that the number of road obstacles emerging in a certain period of observation can be expressed by a negative binomial distribution. Therefore, it is possible to derive readily the indicator for controlling the road obstacle risk.

In the Poisson-Gamma model, the number of road obstacles observed in a certain unit period is expressed by a probability distribution. However, in the data obtained after road patrol, observation period varies according to road sections. Moreover, in order to discuss road patrol frequency, it is necessary to model the effects of patrol cycle on the obstacle emergence risk. In this circumstance, the authors propose a Poisson-Gamma model that explicitly considers patrol cycle (observation period). In addition, in order to secure the operability of the risk control indicators, the arrival rate distribution is expressed by a Gamma distribution with mean 1.

3.2. Formulation of the Model. Assume that the arrival rate of a road obstacle in a road section $i(i=1, \ldots, N)$ is subject to a probability distribution function $F\left(\varepsilon_{i}\right)$, and suppose that the arrival rate $\lambda_{i}>0$ is one actual value. The arrival rate $\lambda_{i}$ is modeled as follows:

$$
\lambda_{i}=\mu_{i} \varepsilon_{i}=\exp \left(x_{i} \beta^{\prime}\right) \varepsilon_{i}
$$

where $\mu_{i}=\exp \left(x_{i} \beta^{\prime}\right)$ is the average arrival rate of road obstacles in road section $i$ and is expressed by using the characteristic $x_{i}$ of road section $i$. In addition, $\varepsilon_{i}$ is the probability error term being subject to a Gamma distribution with mean 1 and variance $\phi^{-1}$. The Gamma distribution is defined in the range $[0, \infty)$, and an exponential function regarding the weighted sum of explanatory variables is used in the righthand side, and so it is guaranteed that with regard to arbitrary explanatory variables and the probability error term, the right-hand side of (1) is positive. Since the mean of the probability error term $\varepsilon_{i}$ is 1 , the expected arrival rate $E\left[\lambda_{i}\right]$ can be expressed by the following equation:

$$
E\left[\lambda_{i}\right]=\exp \left(x_{i} \beta\right)
$$

In general, the probability density function $f\left(\varepsilon_{i}: \alpha, \beta\right)$ of the Gamma distribution $G(\alpha, \beta)$ can be defined as follows:

$$
f\left(\varepsilon_{i}: \alpha, \beta\right)=\frac{1}{\beta^{\alpha} \Gamma(\alpha)} \varepsilon_{i}^{\alpha-1}\left(-\frac{\varepsilon_{i}}{\beta}\right) .
$$

The mean of the Gamma distribution $G(\alpha, \beta)$ is $\mu=\alpha \beta$, and the variance is $\sigma^{2}=\alpha \beta^{2}$. Therefore, the probability density function $f\left(\varepsilon_{i}: \phi, \phi^{-1}\right)$ of the Gamma distribution with mean 1 and variance $1 / \phi$ can be expressed as follows:

$$
f\left(\varepsilon_{i}: \phi, \phi^{-1}\right)=\frac{\phi^{\phi}}{\Gamma(\phi)} \varepsilon_{i}^{\phi-1} \exp \left(-\phi \varepsilon_{i}\right) .
$$

Here, assume that road obstacles emerge with the arrival rate $\lambda_{i}$. At this time, when road patrol is carried out with cycle $z_{i}$, the conditional probability of the discovery of $n_{i}$ road obstacles in road section $i$ can be expressed by the following Poisson distribution:

$$
\operatorname{Po}\left(n\left(z_{i}\right)=n_{i} \mid \lambda_{i}\right)=\frac{\left(\lambda_{i} z_{i}\right)^{n_{i}}}{n_{i} !} \exp \left(-\lambda_{i} z_{i}\right) .
$$

Furthermore, when the arrival rate $\lambda_{i}$ follows the Gamma distribution (4), the marginal probability of the discovery 
of $n_{i}$ road obstacles in road section $i$ with cycle $z_{i}$ can be expressed by the following equation:

$$
\begin{aligned}
P\left(n\left(z_{i}\right)\right. & \left.=n_{i}\right) \\
= & \int_{0}^{\infty} \operatorname{Po}\left(n\left(z_{i}\right)=n_{i} \mid \lambda_{i}\right) f\left(\varepsilon_{i}: \phi, \phi^{-1}\right) d \varepsilon_{i} \\
= & \int_{0}^{\infty} \frac{\phi^{\phi}}{\Gamma(\phi)} \frac{\left(\lambda_{i} z_{i}\right)^{n_{i}}}{n_{i} !} \exp \left(-\lambda_{i} z_{i}\right) \varepsilon_{i}^{\phi-1} \exp \left(-\phi \varepsilon_{i}\right) d \varepsilon_{i} \\
= & \frac{\phi^{\phi}}{n_{i} ! \Gamma(\phi)} \int_{0}^{\infty}\left(\mu_{i} z_{i}\right)^{n_{i}} \varepsilon^{n_{i}+\phi-1} \exp \left\{-\left(\mu_{i} z_{i}+\phi\right) \varepsilon_{i}\right\} d \varepsilon_{i} .
\end{aligned}
$$

Here, when $u_{i}=\left(\mu_{i} z_{i}+\phi\right) \varepsilon_{i}$ is substituted into the equation and the variable of the probability density function is changed, the following expression can be obtained:

$$
\begin{aligned}
\int_{0}^{\infty}\left(\mu_{i} z_{i}\right)^{n_{i}} \varepsilon_{i}^{n_{i}+\phi-1} \exp \left\{-\left(\mu_{i} z_{i}+\phi\right) \varepsilon_{i}\right\} d \varepsilon_{i} \\
=\int_{0}^{\infty} \frac{\left(\mu_{i} z_{i}\right)^{n_{i}}}{\left(\mu_{i} z_{i}+\phi\right)^{n_{i}+\phi}} u_{i}^{\phi+n_{i}-1} \exp \left(-u_{i}\right) d u_{i} \\
=\frac{\Gamma\left(\phi+n_{i}\right)\left(\mu_{i} z_{i}\right)^{n_{i}}}{\left(\mu_{i} z_{i}+\phi\right)^{n_{i}+\phi}} .
\end{aligned}
$$

Therefore, the marginal probability of the discovery of $n_{i}$ $\left(n_{i}=0,1,2, \ldots\right)$ road obstacles in road section $i$ with cycle $z_{i}$ can be expressed by the following equation:

$$
P\left(n_{i}\left(z_{i}\right)=n_{i}\right)=\left(\frac{\phi}{\mu_{i} z_{i}+\phi}\right)^{\phi}\left(\frac{\mu_{i} z_{i}}{\mu_{i} z_{i}+\phi}\right)^{n_{i}} \frac{\Gamma\left(\phi+n_{i}\right)}{n_{i} ! \Gamma(\phi)} .
$$

Hereinafter, the probability distribution model (8) is called the Poisson-Gamma model. In addition, when $p_{i}=\phi /\left(\mu_{i} z_{i}+\right.$ $\phi)$ is substituted, (8) becomes as follows:

$$
\begin{aligned}
P\left(n_{i}\left(z_{i}\right)=n_{i}\right) & =p_{i}^{\phi}\left(1-p_{i}\right)^{n_{i}} \frac{\Gamma\left(\phi+n_{i}\right)}{n_{i} ! \Gamma(\phi)} \\
& =\left(\begin{array}{c}
\phi+n_{i}-1 \\
n_{i}
\end{array}\right) p_{i}^{\phi}\left(1-p_{i}\right)^{n_{i}},
\end{aligned}
$$

where $\Gamma\left(\phi+n_{i}\right)=\left(\phi+n_{i}-1\right) \cdots \phi \Gamma(\phi)$, and it can be expressed as follows:

$$
\left(\begin{array}{c}
\phi+n_{i}-1 \\
n_{i}
\end{array}\right)=\frac{\left(\phi+n_{i}-1\right)\left(\phi+n_{i}-2\right) \cdots \phi}{n_{i} !} .
$$

In addition,

$$
P\left(n_{i}\left(z_{i}\right)=0\right)=p_{i}^{\phi} .
$$

Namely, the Poisson-Gamma model (9) can be expressed as a negative binomial distribution with probability $p_{i}$. In addition, the mean number of road obstacles $E\left[n_{i} \mid z_{i}\right]$ and the variance $V\left[n_{i} \mid z_{i}\right]$ with cycle $z_{i}$ can be expressed by the following equations:

$$
\begin{gathered}
E\left[n_{i} \mid z_{i}\right]=\mu_{i} z_{i}, \\
V\left[n_{i} \mid z_{i}\right]=\frac{\mu_{i} z_{i}\left(\mu_{i} z_{i}+\phi\right)}{\phi} .
\end{gathered}
$$

3.3. Estimation Method of the Model. By conducting road patrol, it is possible to obtain the information on road obstacles. Suppose that a total of $K$ patrol data samples have been obtained after road patrol. The information $e^{j}$ of the patrol sample $j(j=1, \ldots, K)$ is represented by

$$
e^{l}=\left(n^{j}, z^{j}, x_{i(j)}\right)
$$

where $i(j)$ denotes the code number of the target road section $i$ of the data of the patrol sample $j$. In addition, $n^{j}, z^{j}$, and $x_{i(j)}$ represent the number of observed road obstacles, the cycle of road patrol, and the characteristic vector of the road section $i(j)$, respectively.

In the Poisson-Gamma model (9), unknown parameters are $\beta$ and the dispersion parameter is $\phi$. At this time, assume that the actual measurement information $\bar{e}=\left\{\bar{e}^{j}(j=1\right.$, $\ldots, K)\}$ of the patrol sample $j(j=1, \ldots, K)$ has been obtained. The log likelihood function of the Poisson-Gamma model can be expressed by the following equation:

$$
\begin{aligned}
& \ln \{L(\beta, \phi): \bar{e}\} \\
&=\sum_{j=1}^{K}\left\{\ln \left[\frac{\Gamma\left(\phi+\bar{n}^{j}\right)}{\Gamma(\phi)}\right]+\bar{n}^{j} \ln \left(\mu_{i(j)} \bar{z}^{j}\right)\right. \\
&\left.\quad-\left(\bar{n}^{j}+\phi\right) \ln \left(\mu_{i(j)} \bar{z}^{j}+\phi\right)+\phi \ln \phi-\ln \bar{n}^{j} !\right\} .
\end{aligned}
$$

Here, there is the following relation regarding the gamma function:

$$
\ln \left\{\frac{\Gamma\left(\phi+\bar{n}^{j}\right)}{\Gamma(\phi)}\right\}=\sum_{k=0}^{\bar{n}^{j}-1} \ln (\phi+k)
$$

Therefore, the log likelihood function can be converted as follows:

$$
\begin{aligned}
& \ln \{L(\beta, \phi): \bar{e}\} \\
&=\sum_{j=1}^{K}\left\{\sum_{k=0}^{\bar{n}^{j}-1} \ln (\phi+k)+\bar{n}^{j} \ln \left(\mu_{i(j)} \bar{z}^{j}\right)\right. \\
&\left.\quad-\left(\bar{n}^{j}+\phi\right) \ln \left(\mu_{i(j)} \bar{z}^{j}+\phi\right)+\phi \ln \phi-\ln \bar{n}^{j} !\right\},
\end{aligned}
$$

where

$$
\mu_{i(j)}=\exp \left(\bar{x}_{i(j)} \beta^{\prime}\right)
$$

By using the log likelihood function (16), it is possible to obtain the maximum likelihood estimates of the parameters $\beta$ and $\phi$ of the Poisson-Gamma model by means of the maximum-likelihood method. That is, the maximum likelihood estimate of the parameter $\beta$ that maximizes the log 
likelihood function (16) is calculated as the parameter $\hat{\beta}=$ $\left(\hat{\beta}_{1}, \ldots, \hat{\beta}_{M}\right)$ that satisfies the following condition:

$$
\frac{\partial \ln \{L(\hat{\beta}: \bar{e})\}}{\partial \beta_{m}}=0 \quad(m=1, \ldots, M) .
$$

The optimization condition is $M$ th degree simultaneous nonlinear equations, which can be solved by using the sequential iteration method based on the Newton method. Furthermore, the estimator $\widehat{\Sigma}(\hat{\beta})$ of the asymptotic covariance matrix of the parameter can be expressed by the following equation:

$$
\widehat{\Sigma}(\hat{\beta})=\left[\frac{\partial^{2} \ln \{L(\widehat{\beta}: \bar{e})\}}{\partial \beta \partial \beta^{\prime}}\right]^{-1},
$$

where the right-hand side of (19) is the inverse matrix of the $M \times M$ Fisher information matrix whose $(l, m)$ th element is $\partial^{2} \ln \{L(\hat{\beta}: \bar{e})\} / \partial \beta_{l} \partial \beta_{m}$.

\section{Model for Controlling the Risk of Obstacle Emergence}

4.1. Purpose in Risk Management. As the indicator for risk management, the authors propose the number of road obstacles. The risk management indicator can be defined for each road section or each route. Here, the risk management indicators are defined for each road section $i(i=1, \ldots, I)$. Next section defines the distribution of the number of road obstacles discovered during road patrol, while defining the expectation of the number of discovered road obstacles and the $\mathrm{VaR}$ index. If the emergence of road obstacles follows a probabilistic process, the number of road obstacles discovered during road patrol is subject to a probability distribution. In the case where risk management about the number of discovered road obstacles is conducted under a certain confidence level, it is necessary to introduce the VaR index that takes into account the variance of the number of discovered road obstacles. The above can be discussed with a statistic method using the mixture Poisson process model. However, some road sections have outstanding risk of road obstacle emergence due to the local road condition or environmental condition, compared with the statistical risk level. For coping with such road sections, it is necessary to conduct intensive risk management, such as the installation of monitoring cameras. For controlling the risk of road obstacle emergence, it is also important to extract such road sections that need intensive risk management. This issue is also discussed in this chapter.

4.2. Number of Road Obstacles. Suppose that patrol is conducted on the road section $i(i=1, \ldots, I)$ at the $z_{i}$ intervals. At this time, the probability of the discovery of $n_{i}\left(n_{i}=0\right.$, $1, \ldots)$ road obstacles can be expressed by the following negative binomial distribution, based on (9). The expected number $E\left[n_{i} \mid z_{i}\right](12 \mathrm{a})$ is an intuitively understandable index. However, the expected number is defined as the expectation of the number of road obstacles observed during repeated patrol, and this number does not mean the number of actually observed road obstacles. It is probable that the number of road obstacles observed in each patrol cycle will exceed $E\left[n_{i} \mid\right.$ $z_{i}$ ]. In order to control the road obstacle risk, it is desirable to use a management indicator that can explicitly consider the probability distribution of the number of road obstacles. Accordingly, let us formulate the VaR (Value at Risk) index as an indicator for controlling the risk of road obstacle emergence. Letting $z_{i}$ represent the patrol cycle length, the probability that the number of road obstacles observed during patrol will exceed an allowable level (hereinafter called risk management limit) $\bar{U}_{i}$ can be expressed by the following equation:

$$
P\left(n_{i} \geq \bar{U}_{i} \mid z_{i}\right)=\sum_{n_{i}=\left[\bar{U}_{i}\right]}^{\infty} \mathrm{NB}\left(n_{i}: z_{i}\right),
$$

where $\left[\bar{U}_{i}\right]$ represents the smallest integer among the integers larger than $\bar{U}_{i}$. Figure 2 shows the probability distribution of the number of road obstacles, the expected number of road obstacles, and the risk management limit. The number of obstacles, which is the random variable of this case, is discrete, and so the sum $\omega$ of the length of line segments (probabilities) in the region of $n_{i} \geq \alpha$ in the figure (dashed lines) means the probability that the number of road obstacles observed during patrol exceeds the specified risk management limit: $\bar{U}_{i}=\alpha$. Since there is uncertainty in the process of the emergence of road obstacles, the number of road obstacles observed during patrol does not always meet a specified management limit. The probability $\omega$ is the index indicating the risk of obstacle emergence and called the obstacle emergence confidence level. Here, let the following equation define the VaR index regarding the number of road obstacles:

$$
\operatorname{VaR}_{\omega}^{\alpha}\left(z_{i}\right)=\arg \max _{U_{i}}\left\{U_{i} \mid P\left(n_{i} \geq U_{i} \mid z_{i}\right) \leq \omega\right\},
$$

where $\omega$ is the obstacle emergence confidence level, $z_{i}$ is the patrol cycle, and arg is the symbol for specifying $U_{i}$ that maximizes the right-hand side of (21). In addition, the superscript $\alpha$ indicates the VaR index regarding the number of road obstacles. Here, the set $\Omega_{\omega}\left(\bar{U}_{i}\right)$ is defined as follows:

$$
\Omega_{\omega}\left(\bar{U}_{i}\right)=\left\{z_{i} \mid \operatorname{VaR}_{\omega}^{\alpha}\left(z_{i}\right) \leq \bar{U}_{i}\right\} .
$$

The set $\Omega_{\omega}\left(\bar{U}_{i}\right)$ represents "the set of patrol cycle with which it is possible to keep the number of road obstacles below the risk management limit $\bar{U}_{i}$ with the obstacle emergence confidence level $\omega$." Like this, the risk of road obstacle emergence can be expressed by using the two parameters: the confidence level $\omega$ and the risk management limit $\bar{U}_{i}$. The confidence level corresponds to the significance level in statistics, and $\omega=0.05$ or 0.01 is adopted in general. For the risk management limit, unacceptable specific quantity (the number of obstacles in this case) is specified. Needless to say, lowering the confidence level or risk management limit means adopting a stricter road patrol policy. Actually, road administrators can determine the optimum patrol cycle with 


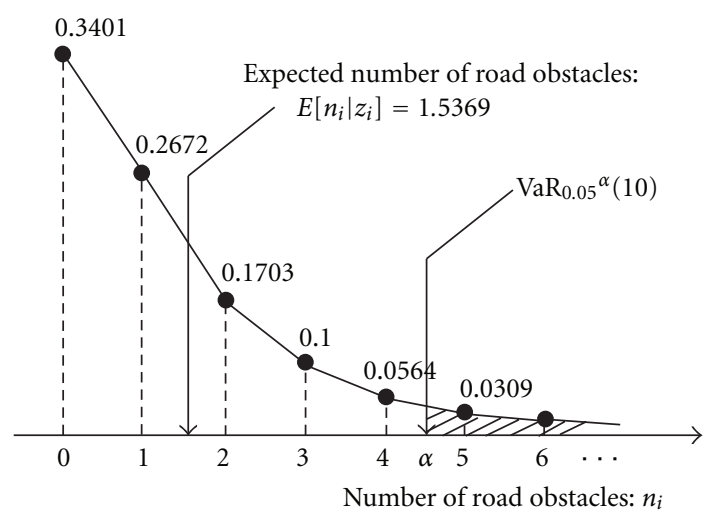

Figure 2: Distribution of road obstacle emergence.

the above equation, by specifying the confidence level and risk management limit. The expected number $E\left[n_{i} \mid z_{i}\right]$ is identical to the VaR value $\operatorname{VaR}_{0.5}^{\alpha}\left(z_{i}\right)$ whose confidence level is equal to 0.5 . According to the definition of the VaR index, when $\omega<0.5, E\left[n_{i} \mid z_{i}\right]<\operatorname{VaR}_{\omega}^{\alpha}\left(z_{i}\right)$.

4.3. Extraction of Intensive Management Sections. Suppose that road patrol is carried out $L_{i}$ times with the cycle $z_{i}$, for the road section $i(i=1, \ldots, I)$. Let $n_{i}^{l_{i}}$ represent the number of road obstacles discovered during the $l_{i}$ th road patrol $\left(l_{i}=1, \ldots, L_{i}\right)$. In addition, let $n_{i}=\left(n_{i}^{1}, \ldots, n_{i}^{L_{i}}\right)$ denote the observation sample vector regarding the number of road obstacles discovered in the $L_{i}$-time patrol. At this time, there is a possibility that the number of road obstacles will be larger than the average number of road obstacles estimated by the Poisson-Gamma model. In a road section where such an abnormal value is obtained, the road environment condition unique to said road section may influence the risk of obstacle emergence. For such road sections, it is necessary to conduct not only daily road patrol but also intensive road obstacle management. In this study, a road section where the arrival rate of road obstacles is abnormally high is called an intensive management section. In order to extract intensive management sections, it is necessary to check whether the arrival rate of road obstacles in the population of the target road sections is significantly different from the arrival rate in the extracted road section. The discussion on the difference between a specific sample and the reference population in probability characteristics has been well developed as the credibility theory in the field of non-life-insurance theory. This study evaluates the necessity of the intensive management of road sections, with a statistical method based on the credibility theory.

As mentioned above, in the road section having the characteristic $x_{i}$ with the patrol cycle $z_{i}$, the expected number of road obstacles $E\left[n_{i} \mid z_{i}\right]$ can be expressed by the following equation:

$$
E\left[n_{i} \mid z_{i}\right]=\exp \left(x_{i} \beta^{\prime}\right) z_{i}
$$

On the other hand, the sample mean $\bar{X}_{i^{*}}$ is defined as follows, using the number of road obstacles $n_{i^{*}}^{l_{*}^{*}}\left(l_{i^{*}}=1, \ldots, L_{i^{*}}\right)$ measured in the $L_{i}$-time patrols in the target road section $i^{*}$ :

$$
\bar{X}_{i^{*}}=\frac{\sum_{l^{*}=1}^{L_{i^{*}}} n_{i^{*}}^{l_{i *}}}{L_{i^{*}}} .
$$

At this time, there is the following relation with regard to the sample mean $\bar{X}_{i^{*}}$ :

$$
E\left[\bar{X}_{i^{*}}\right]=L_{i^{*}}^{-1} \sum_{l_{i^{*}=1}}^{L_{i^{*}}} E\left[n_{i^{*}}^{l_{i *}^{*}} \mid z_{i^{*}}\right]=E\left[n_{i^{*}} \mid z_{i^{*}}\right] .
$$

Therefore, it can be understood that the sample mean $\bar{X}_{i^{*}}$ is identical to the unbiased estimator of $E\left[n_{i^{*}} \mid z_{i^{*}}\right]$. The sample variance also can be expressed as follows, using the variance $V\left[n_{i} \mid z_{i}\right]$ of the negative binomial distribution in the same way:

$$
V\left[\bar{X}_{i^{*}}\right]=L_{i^{*}}^{-2} \sum_{i_{i^{*}}=1}^{L_{i^{*}}} V\left[n_{i^{*}}^{l_{i *}} \mid z_{i^{*}}\right]=L_{i^{*}}^{-1} V\left[n_{i^{*}} \mid z_{i^{*}}\right] .
$$

These two equations indicate that as the number of times of patrol $L_{i^{*}}$ becomes somewhat large, the variance of the sample mean $\bar{X}_{i^{*}}$ approaches 0 and $\bar{X}_{i^{*}}$ converges to $E\left[n_{i^{*}} \mid z_{i^{*}}\right]$, making stochastic convergence. Therefore, it is possible to statistically judge whether or not road obstacles emerge while following the Poisson-Gamma model, that is, whether or not road obstacles emerge in a normal manner, by specifying a probability distribution of the sample mean and evaluating whether the predetermined risk level $p$ (e.g., $p=0.9$ ) satisfies the following relation:

$$
\operatorname{Pr}\left(\left|\bar{X}_{i^{*}}-E\left[n_{i^{*}} \mid z_{i^{*}}\right]\right| \leq y\left(i^{*}\right)\right) \geq p .
$$

Here, the central limit theorem guarantees that the probability distribution of the sample mean $\bar{X}_{i^{*}}$ becomes a normal distribution as a whole when the number of times of patrol is large. Furthermore, when the normalization constant $Z$ is defined as follows:

$$
Z=\frac{\bar{X}_{i^{*}}-E\left[n_{i^{*}} \mid z_{i^{*}}\right]}{\left(\sqrt{L_{i^{*}}}\right)^{-1} V\left[n_{i^{*}} \mid z_{i^{*}}\right]},
$$

the normalization constant $Z$ is subject to the standard normal distribution $N(0,1)$. Therefore, (27) can be expressed as follows:

$$
\begin{array}{r}
\operatorname{Pr}\left(|Z| \leq \frac{y\left(i^{*}\right)}{\left(L_{i^{*}}\right)^{-1} V\left[n_{i^{*}} \mid z_{i^{*}}\right]}\right) \\
=\operatorname{Pr}\left(|Z| \leq y_{p}\left(i^{*}\right)\right) \geq p .
\end{array}
$$

The cumulative distribution function of the standard normal distribution is available in the form of a table of figures in a lot of literatures, and so it is possible to uniquely specify $y_{p}\left(i^{*}\right)$ in the above equation. When the following condition is not satisfied with a given risk level $p$,

$$
|Z| \leq y_{p}\left(i^{*}\right)
$$

it can be concluded that the road section concerned has outstanding characteristic regarding the emergence of road obstacles compared with other road sections. 
TABLE 1: Route data.

\begin{tabular}{lccc}
\hline Route & A1 and A2 & B1 and B2 & C1 and C2 \\
\hline Route length $[\mathrm{km}]$ & 82.6 & 88.3 & 93.6 \\
Number of sample & 3,820 & 3,296 & 4,140 \\
Number of fallen objects & 3,501 & 3,079 & 3,817 \\
Number of average fallen objects & 8.48 & 6.97 & 8.16 \\
\hline
\end{tabular}

\section{Empirical Study}

5.1. Outline of the Application Cases. In this study, the authors analyze the database of road patrol conducted on general national road, Route A (target section length: $82.6 \mathrm{~km})$, Route B $(88.3 \mathrm{~km})$, and Route C $(93.6 \mathrm{~km})$. This database has accumulated the data of daily and night-time patrols from April 1, 2009 to March 31, 2010. The daily logs of road patrol record not only the type and number of road obstacles but also the routes and road sections where road obstacles were discovered and the patrol times. As tabulated in Table 1, the three routes, which are the targets of the empirical analysis, are composed of 413, 442, and 468 unit sections, respectively. Here, the length of one road section is $200 \mathrm{~m}$. In addition, as concrete road obstacles, fallen objects and pavement abnormalities are focused on. The road obstacles that emerged on the three national routes during the analysis-target period include $3,501,3,079$, and 3,817 samples on each route.

5.2. Estimation of the Poisson-Gamma Model. In order to model the emergence processes of road obstacles, the Poisson-Gamma model was estimated. For this estimation, 11,256 sample data of normal patrol were available. As explanatory variables, the following parameters were adopted. Namely, the estimation equation can be expressed as follows:

$$
\lambda_{i}=\exp \left(\beta_{1}+\beta_{2} x_{i}^{2}+\beta_{3} x_{i}^{3}+\beta_{4} x_{i}^{4}+\beta_{i} x_{i}^{5}\right) \varepsilon_{i}
$$

where $\beta_{1}, \beta_{2}, \beta_{3}, \beta_{4}, \beta_{5}$ represent parameters, $x_{i}^{2}$ represents large-size automobile traffic volume in the day time, $x_{i}^{3}$ denotes travel speed, $x_{i}^{4}$ represents the average amount of rutting depth, and $x_{i}^{5}$ represents roadside classification. $\beta_{1}$ is a constant term. In this case, the arrival rate $\lambda_{i}$ of the emergence of road obstacles is composed of the common characteristic $\beta_{1}$ in all road sections, the common characteristic $\beta_{2} x_{i}^{2}+\beta_{3} x_{i}^{3}+\beta_{4} x_{i}^{4}+\beta_{i} x_{i}^{5}$ in the same environmental condition, and the heterogeneous characteristic $\varepsilon_{j}$ of each road section. The first-order optimization condition of (18) regarding the Poisson-Gamma model is given as simultaneous nonlinear equations, and the maximum likelihood estimator of unknown parameters was calculated with the Newton-Raphson method. The estimation results and $t$-value are shown in Table 2. This table tabulates the estimation results.

Let us obtain the cumulative discovery probability of road obstacles in target area of Routes A, B, and C, using the above-estimated Poisson-Gamma model. By analyzing the relation between the cumulative discovery probability and the patrol cycle, it is possible to analyze how the

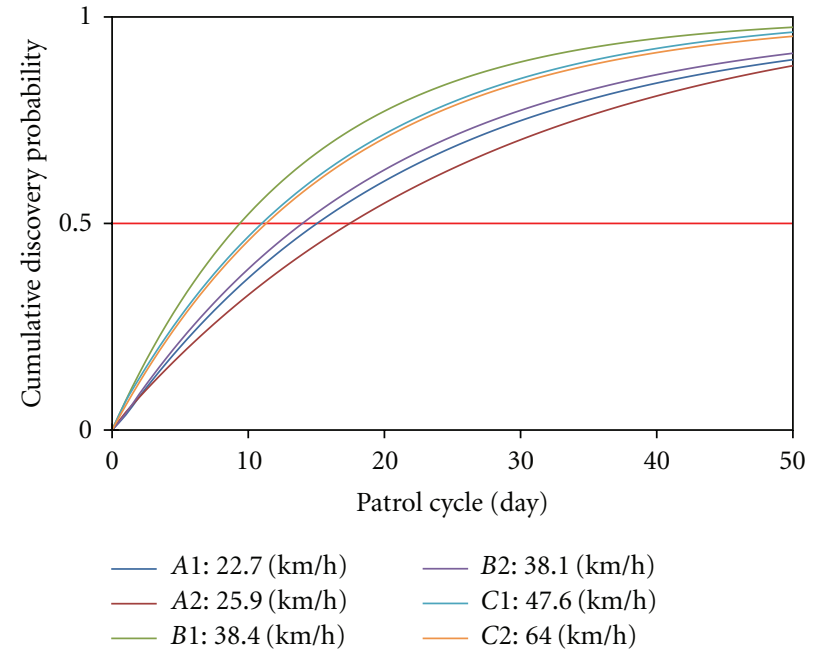

FIGURe 3: Cumulative discovery probability in the Poisson-Gamma model.

probability of the discovery of road obstacles in the route or road section concerned changes due to the patrol cycle. The cumulative discovery probability can be obtained by calculating the probability of the discovery of one or more road obstacles under a given patrol cycle. This is equivalent to the subtraction of the probability that road obstacles do not emerge from the total probability 1. Namely, the cumulative probability of the discovery of road obstacles can be defined as follows:

$$
P\left(n_{i}\left(z_{i}\right) \geq 1\right)=1-\prod_{i=1}^{I} P\left(n_{i}\left(z_{i}\right)=0\right) .
$$

The distribution of the number of road obstacles based on the Poisson-Gamma model can be expressed by (8). Therefore, the cumulative emergence probability of road obstacles under the Poisson-Gamma model can be expressed by the following equation:

$$
P_{\mathrm{NB}}\left(n_{i}\left(z_{i}\right) \geq 1\right)=1-\prod_{i=1}^{I}\left(\frac{\phi}{\mu_{i} z_{i}+\phi}\right)^{\phi} .
$$

Figure 3 shows the results of the analysis of the relation between patrol cycle and the cumulative discovery probability of road obstacles based on the estimated PoissonGamma model. Each characteristic parameter is adopted median value. As for travel speed, its median values are shown in the figure. The advantage in adopting the PoissonGamma model is that it is possible to estimate the risk of road obstacle emergence explicitly, using the variance parameter $\phi$ by taking into account the variance of arrival rate.

5.3. Analytical Results. In order to determine the optimum patrol cycle of the target route for road patrol, the risk management indicator for the patrol cycle mentioned and the optimum patrol cycle for the risk management limit are calculated for all road sections. 
TABLe 2: Estimation results of the Poisson-Gamma model.

\begin{tabular}{|c|c|c|c|c|c|c|}
\hline & \multicolumn{6}{|c|}{ Poisson-Gamma model } \\
\hline & $\beta_{1}$ & $\beta_{2}$ & $\beta_{3}$ & $\beta_{4}$ & $\beta_{5}$ & $\phi$ \\
\hline Maximum likelihood estimate & -4.82 & $8.51 \times 10^{-5}$ & $1.57 \times 10^{-2}$ & $9.49 \times 10^{-3}$ & 0.11 & 3.20 \\
\hline$(t$-value $)$ & $(-15.51)$ & $(14.56)$ & $(17.25)$ & $(4.22)$ & $(3.99)$ & $(20.22)$ \\
\hline Log likelihood & \multicolumn{6}{|c|}{$-18,325$} \\
\hline AIC & \multicolumn{6}{|c|}{36,663} \\
\hline
\end{tabular}

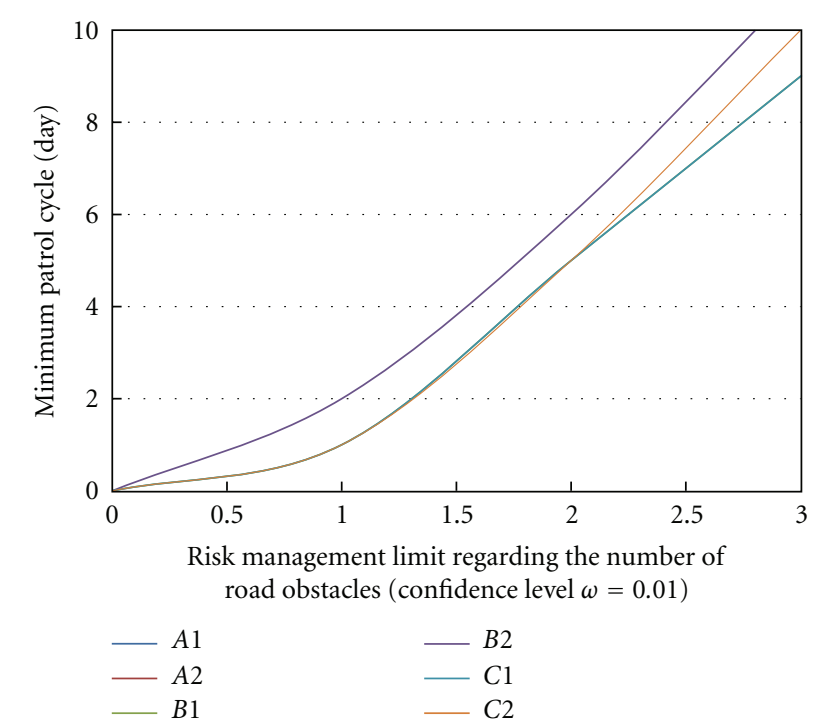

Figure 4: Risk management limit (number of road obstacles) and the minimum patrol cycle (confidence level $\omega=0.01$ ).

Firstly, the VaR index regarding the number of road obstacles $\operatorname{VaR}_{\omega}^{\alpha}\left(z_{i}\right)$ (refer to (21)) was obtained, based on the Poisson-Gamma model. The VaR index can be calculated for all road sections, but the volume of the results becomes enormous, and so the authors target the case of a certain road section, where the number of road obstacles is relatively large in each section.

Figures 4 and 5 show the relation between the risk management limit $\bar{U}_{i}$ regarding the number of road obstacles and the minimum patrol cycle defined as the minimum value of the set $\Omega_{\omega}\left(\bar{U}_{i^{\circ}}\right)$ (refer to (22)). Figure 4 is focused on that. In route $\mathrm{B} 2$, if the confidence level is set as $\omega=0.01$ and the risk management limit is set to be 1 , though overlap lines in the graph, the minimum patrol cycle becomes 2 days. Next, Figure 5 is focused on that. In route B2, if the confidence level is set as $\omega=0.05$ and the risk management limit is set to be 1 , though overlap lines in the graph, the minimum patrol cycle becomes 5 days.

The above passages discussed the minimum patrol cycle that satisfies the risk management limit for each road section, for the proposed risk management indicator. It can be inferred that which is adopted as a risk management indicator depends on the situation of the roads managed by the road administrator concerned, but it can be considered that the proposed indicator is practical for gauging the risk management limit for road obstacles. In addition, even if

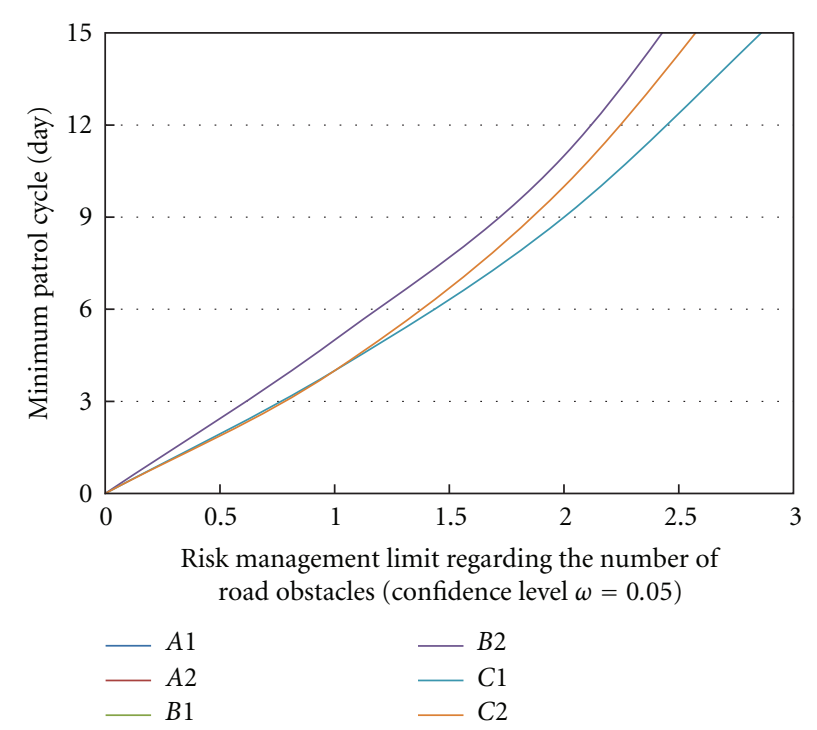

Figure 5: Risk management limit (number of road obstacles) and the minimum patrol cycle (confidence level $\omega=0.05$ ).

another indicator is adopted, the risk management limit can be defined by adopting the same idea.

\section{Conclusion}

In this study, the authors have proposed a method for evaluating the risk of emergence of road obstacles, including fallen objects, road surface deformation, damages to roadattached facilities, and also a methodology for designing a road patrol policy that can curtail the patrol costs effectively. Through this study, the authors also proposed a method of expressing the risk of emergence of road obstacles based on the Poisson-Gamma model. In addition, for the risk management of road obstacles, the authors pointed out that the proposed indicator is important. Furthermore, the authors studied the case of application to national routes and empirically clarified that it is effective to adopt a mixture Poisson process model that takes into account the heterogeneity in the arrival rates of road obstacles, in order to describe the emergence processes of actual road obstacles. In addition, the authors designed a patrol policy that can appropriately control the obstacle emergence risk with a limited amount of budget, using the obstacle emergence risk management model proposed in this study.

The methodology proposed in this study is highly practical, but there still remain the following problems to be solved. The first problem is that the analysis target in this 
study was limited to specific national routes. In order to study a variety of road characteristic variables, it is essential to collect patrol data of a broad range of routes and accumulate the cases of application of the proposed methodology. The second problem is that this study assumed that the length of patrol cycle does not influence the probability distribution of arrival rates. There is a possibility that the emergence risk of road obstacles will depend on road patrol policy according to patrol cycle and road characteristics. One possible method for coping with such problems is to estimate the variance parameter $\phi$ of the Poisson-Gamma model while defining a road patrol measure or road characteristic as an explanatory variable. Lastly, it is necessary to discuss the method for specifying the risk management limit. For instance, it is necessary to glean information for specifying a desirable risk confidence level, by analyzing the relation between the road obstacle risk and the number of defective accidents, complaints from residents and users, and so forth. In addition, the method proposed in this study is applicable to the designing of patrol policy for not only road facilities but also other transportation facilities. In this case, the primary discussion theme would be the setting of a risk management limit in accordance with the characteristics of each transportation facility.

\section{Acknowledgment}

For conducting this study, the authors received a great deal of support; for example, some data was provided by the Road Administration Section, Kinki Regional Development Bureau, the Ministry of Land, Infrastructure and Transport.

\section{References}

[1] D. Lord, "Modeling motor vehicle crashes using Poisson-gamma models: examining the effects of low sample mean values and small sample size on the estimation of the fixed dispersion parameter," Accident Analysis \& Prevention, vol. 38, no. 4, pp. 751-766, 2006.

[2] J. A. McNeil, R. Frey, and P. Embrechts, Quantitative Risk Management, Princeton University Press, Princeton, NJ, USA, 2005.

[3] R. A. Fisher, "The negative binomial distribution," Annals of Eugenics, vol. 11, pp. 182-187, 1941.

[4] F. J. Anscombe, "Sampling theory of the negative binomial and logarithmic series distribution," Biometrika, vol. 36, pp. 358$382,1950$.

[5] L. R. Shenton and P. A. Wallington, "The bias of moment estimators with an application to the negative binomial," Biometrika, vol. 49, no. 1-2, pp. 193-204, 1962.

[6] A. C. Cameron and P. K. Trivedi, "Regression-based tests for overdispersion in the Poisson model," Journal of Econometrics, vol. 46, no. 3, pp. 347-364, 1990.

[7] A. C. Cameron and P. K. Trivedi, Regression Analysis of Count Data, Cambridge University Press, Cambridge, UK, 1998. 

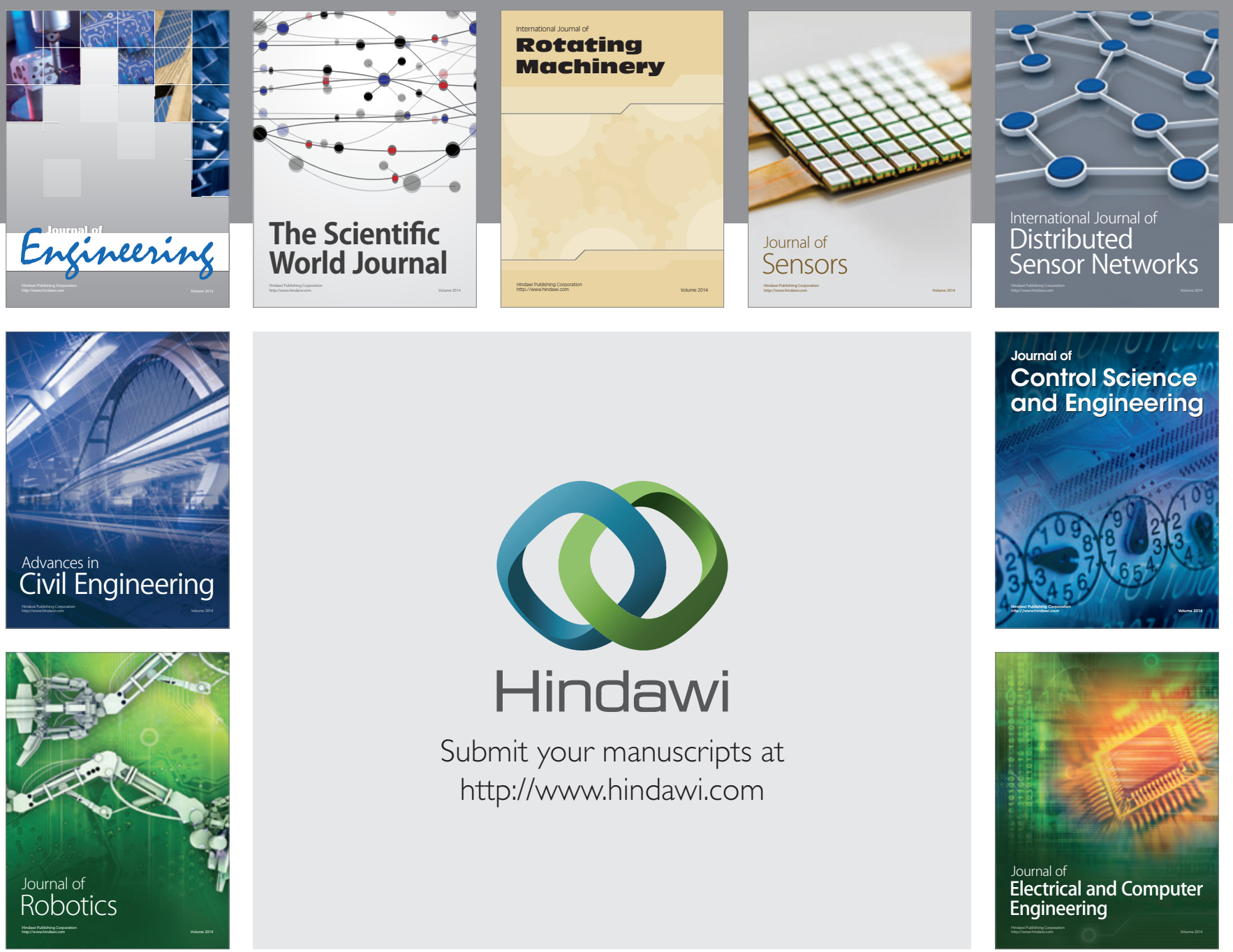

Submit your manuscripts at

http://www.hindawi.com
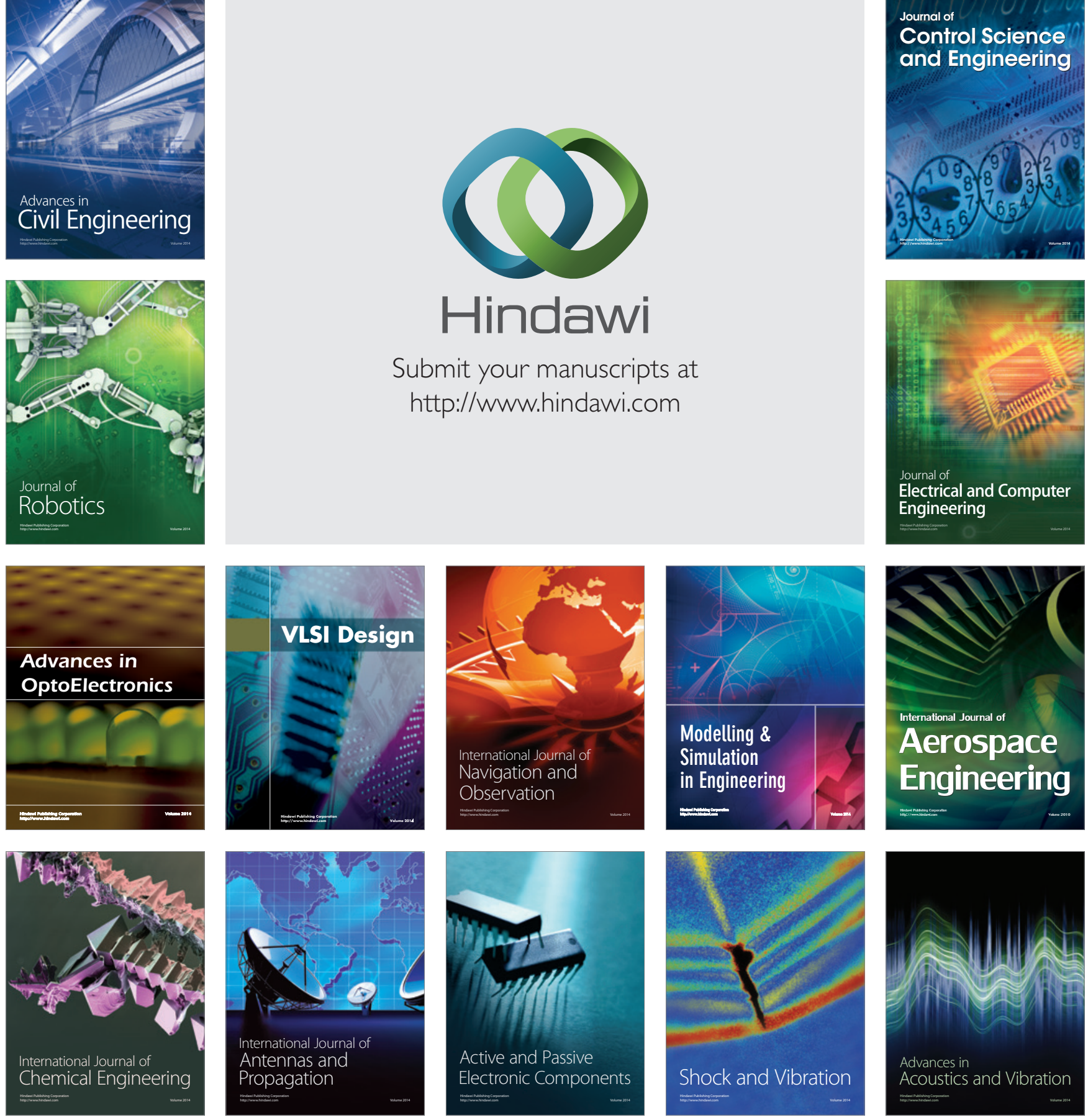